\title{
Solar and Interplanetary Magnetic Helicity Balance of Active Regions
}

\author{
Cristina H. Mandrini \\ Instituto de Astronomía y Física del Espacio, CC.67, Suc.28, 1428, \\ Buenos Aires, Argentina
}

Pascal Démoulin

1. Observatoire de Paris, LESIA, F-92195, Meudon, France

Lidia van Driel-Gesztelyi ${ }^{1,2,3}$

2. Konkoly Observatory, H-1525 Budapest, P.O. Box 67, Hungary

3. Mullard Space Science Laboratory, UCL, Holmbury St. Mary, Dorking, Surrey, RH5 6NT, UK

Sergio Dasso

Instituto de Astronomía y Física del Espacio, CC.67, Suc.28, 1428, Buenos Aires, Argentina

Lucinda M. Green

Mullard Space Science Laboratory, UCL, Holmbury St. Mary, Dorking, Surrey, RH5 6NT, UK

Marcelo López Fuentes

Naval Research Laboratory, Code 7675, Washington,DC 20375-5352

\begin{abstract}
We analyzed the long-term evolution of two active regions (ARs), NOAA 7978 and 8100 , from their emergence through their decay using observations from several instruments on board SoHO (MDI, EIT and LASCO) and Yohkoh/SXT. We computed the evolution of the relative coronal magnetic helicity from one central meridian passage to the next, combining data from MDI and SXT with linear force-free models of the coronal magnetic field. Next, we calculated the injection of helicity by photospheric differential rotation using MDI magnetic maps and a mean differential rotation profile. To estimate the depletion of magnetic helicity we counted all the CMEs of which these ARs were the source, and we evaluated their helicity assuming a one to one correspondence with magnetic clouds (MCs) with an average helicity content; this value was computed for a sample of 18 clouds using a cylindrical linear force-free model. Out of our three helicity estimates (variation of coronal magnetic helicity, injection by differential rotation and ejection via CMEs) the one with the largest uncertainty is the amount of helicity ejected via CMEs. However, we determined, by modeling a particular MC using three different approaches in cylindrical geometry (two force-free models and a non force-free model with constant current), that its magnetic helicity content was nearly independent of the model used to fit in situ field observations (Dasso et al. 2003, in preparation). This result justi-
\end{abstract}


fies our use of the average magnetic helicity value considering only a single MC model. Comparing the three components in the helicity balance (see Table 1), we find that photospheric differential rotation is a minor contributor to the AR magnetic helicity budget. CMEs carry away at least 10 times more helicity than the one differential rotation can provide. Therefore, the magnetic helicity flux needed in the global balance should come from localized photospheric motions that, at least partially, reflect the emergence of twisted flux tubes. Taking into account the magnetic flux in the ARs and the number of turns that a uniformly twisted flux tube should have to survive its rise through the convection zone, we have found that the total helicity carried away in CMEs is approximately equal to the end-to-end helicity of the flux tubes that formed these two ARs. Therefore, we conclude that most of the helicity ejected in CMEs is generated below the photosphere and emerges with the magnetic flux. Extended versions of this work were published in Démoulin et al. (2002, Astronomy \& Astrophys. 382, 650) and in Green et al. (2002, Solar Phys. 208, 43), while in Mandrini et al. (2003, Astrophys. \& Space Sci., 290, 319) and van Driel-Gesztelyi et al. (2003, Adv. Space Res., 32, 1855) the helicity computations were revised to include the underestimation of magnetic flux density found in MDI data. After this revision, we confirmed our former results.

Table 1. The total magnetic helicity balance of AR 7978 and AR 8100 along five and four solar rotations, respectively. $\Delta H_{\text {cor. }}$ and $\Delta H_{\text {d.r. }}$ are the coronal helicity variation and the helicity injected by differential rotation, respectively. The helicity in MCs $\left(\Delta H_{\text {m.cl. }}\right)$ is given taking two extreme lengths ( 0.5 and 2 $\mathrm{AU}$ ) and using the observed and corrected CME numbers (corrections done for gaps and far-side locations). All values are in units of $10^{42} \mathrm{Mx}^{2}$.

\begin{tabular}{lcrcc}
\hline $\begin{array}{l}\text { Active } \\
\text { Region }\end{array}$ & $\Delta H_{\text {cor. }}$ & $\Delta H_{\text {d.r. }}$ & $\begin{array}{c}\Delta H_{\text {m.cl. }} \\
\text { (CME obs. })\end{array}$ & $\begin{array}{c}\Delta H_{\text {m.cl. }} \\
\text { (CME cor. })\end{array}$ \\
\hline NOAA 7978 & -11.0 & 16.2 & {$[36 ., 144]$.} & {$[40 ., 160]$.} \\
NOAA 8100 & -38.8 & -14.6 & {$[38 ., 152]$.} & {$[82 ., 328]$.}
\end{tabular}

Acknowledgments. The authors thank the SOHO/MDI consortium for SOHO data and the MSSL/UCL Solar UK Research Facility for Yohkoh data. SOHO is a joint project by ESA and NASA. L.v.D.G. acknowledges the Hungarian government grant OTKA T-038013. C.H.M. and L.v.D.G. acknowledge financial support from TET (Hungary) and SECyT (Argentina) through their cooperative science program (AR03/02-HU/A01/UIII/01). P.D. and C.H.M. acknowledge support from ECOS (France) and SECyT through their cooperation agreement (A01U04). 